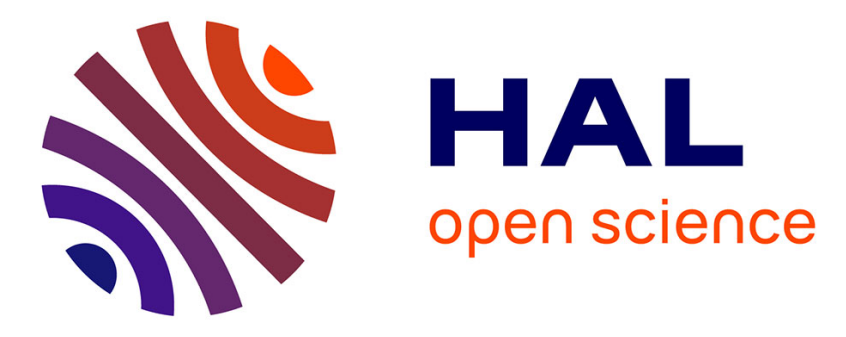

\title{
Nanoscale mechanisms for the reduction of heat transport in bismuth
}

\author{
Maxime Markov, Jelena Sjakste, Giorgia Fugallo, Lorenzo Paulatto, Michele \\ Lazzeri, Francesco Mauri, Nathalie Vast
}

\section{- To cite this version:}

Maxime Markov, Jelena Sjakste, Giorgia Fugallo, Lorenzo Paulatto, Michele Lazzeri, et al.. Nanoscale mechanisms for the reduction of heat transport in bismuth. Physical Review B: Condensed Matter and Materials Physics (1998-2015), 2016, 10.1103/PhysRevB.93.064301 . hal-01284828

\section{HAL Id: hal-01284828 \\ https://hal.science/hal-01284828}

Submitted on 16 Mar 2016

HAL is a multi-disciplinary open access archive for the deposit and dissemination of scientific research documents, whether they are published or not. The documents may come from teaching and research institutions in France or abroad, or from public or private research centers.
L'archive ouverte pluridisciplinaire HAL, est destinée au dépôt et à la diffusion de documents scientifiques de niveau recherche, publiés ou non, émanant des établissements d'enseignement et de recherche français ou étrangers, des laboratoires publics ou privés. 


\title{
Nanoscale mechanisms for the reduction of heat transport in bismuth
}

\author{
Maxime Markov, ${ }^{1}$ Jelena Sjakste, ${ }^{1}$ Giorgia Fugallo, ${ }^{1}$ Lorenzo Paulatto, ${ }^{2}$ Michele Lazzeri, ${ }^{2}$ \\ Francesco Mauri, ${ }^{3}$ and Nathalie Vast ${ }^{1, *}$ \\ ${ }^{1}$ École Polytechnique, Laboratoire des Solides Irradiés, CNRS UMR 7642, CEA-DSM-IRAMIS, \\ Université Paris-Saclay, F91128 Palaiseau cedex, France \\ ${ }^{2}$ IMPMC, UMR CNRS 7590, Sorbonne Universités - UPMC Univ. Paris 06, MNHN, IRD, 4 Place Jussieu, F-75005 Paris, France \\ ${ }^{3}$ Dipartimento di Fisica, Università di Roma La Sapienza, Piazzale Aldo Moro 5, I-00185 Roma, Italy
}

(Received 7 September 2015; published 1 February 2016)

\begin{abstract}
Hand-on routes to reduce lattice thermal conductivity (LTC) in bismuth have been explored by employing a combination of Boltzmann's transport equation and ab initio calculations of phonon-phonon interaction within the density functional perturbation theory. We have first obtained the temperature dependence of the bulk LTC in excellent agreement with available experiments. A very accurate microscopic description of heat transport has been achieved and the electronic contribution to thermal conductivity has been determined. By controlling the interplay between phonon-phonon interaction and phonon scattering by sample boundaries, we predict the effect of size reduction for various temperatures and nanostructure shapes. The largest heat transport reduction is obtained in polycrystals with grain sizes smaller than $100 \mathrm{~nm}$.
\end{abstract}

DOI: 10.1103/PhysRevB.93.064301

\section{INTRODUCTION}

Bismuth is an important semimetallic material for applications of the thermoelectric effect because of the low thermal conductivity (TC) [1]. Indeed, the thermoelectric efficiency $Z T$ is inversely proportional to the thermal conductivity. It is however also proportional to the power factor $\sigma S^{2}$, with $\sigma$ the electrical conductivity and $S$ the Seebeck coefficient. The Seebeck effect (S) is large in bismuth, but the presence of two types of carriers, electrons and holes, at the same time and in low concentrations, is not favorable [2] for $\sigma$.

Several experimental routes have been proposed to obtain one single type of carrier in bismuth, like doping the bulk material with a few percents of antimony to favor electron conduction with respect to hole transport $[3,4]$; or applying a magnetic field to promote field-induced hole Landau states at the Fermi level [5]. Alternatively, nanostructuring on the scale of a few tens of nanometers, with the aim of opening a confinement-induced energy gap [6-8], allows one to break the electron-hole symmetry around the Fermi level [2]. This has led to an increase of $S$ by several orders of magnitude in nanowires $[8,9]$. The latter route is a very appealing concept for a bismuth-based thermoelectric device because it enables either $n$ - or $p$-type doping, allowing one to use the same material in the two legs of the thermocouple [10].

In contrast to $\mathrm{Si}$ [11-16] and $\mathrm{SiGe}$ alloys [12,17], systematic studies of the nanostructuring effect on the thermal conductivity are scarce in bismuth. A quasisuppression of the thermal conductivity has been found in bismuth nanowires, but its origin is still debated [2]. Measurements made on thin films [18-20] and nanowires [2,21-25], most of which are semiconducting, have yielded widely scattered values, preventing a deep understanding of the mechanisms at play in nanostructures [2]. A theoretical determination of the nanostructuring effect on the lattice thermal conductivity (LTC) of bismuth has thus become mandatory.

\footnotetext{
*nathalie.vast@polytechnique.edu
}

In bulk crystal, because of the uniaxial symmetry, the transport is anisotropic and is completely determined only by measuring the LTC in two directions, along the trigonal axis and perpendicular to it - either the binary or the bisectrix directions. Data are missing even in bulk bismuth: in the trigonal direction, the lattice conductivity has not been determined; in the binary direction, Uher et al. [26] were able to measure both total and electron and hole contributions solely up to the temperature $T \approx 150 \mathrm{~K}$. Estimations of the lattice part of the thermal conductivity given in experimental works [26,27] do not agree with each other, nor with the theoretical work of Ref. [28]. At ambient temperature, Gallo et al. (indirectly) measured 1.7 and $0.9 \mathrm{~W}(\mathrm{~K} \mathrm{~m})^{-1}$ in the binary and trigonal directions, respectively [27], while the theoretical values produced by recent ab initio calculations [28] were 4.4 and $3.9 \mathrm{~W}(\mathrm{~K} \mathrm{~m})^{-1}$. Thus large uncertainties also exist on the absolute value of the LTC in bulk Bi.

In this work, we show that $a b$ initio calculations of the phonon-phonon interaction [29] and of phonon scattering by sample boundaries, combined to a transport equation for the phonon system, namely the Boltzmann transport equation (BTE) linearized to first order in the phonon distribution [30] $N$, reliably predicts the LTC for bulk bismuth down to temperatures as low as $30 \mathrm{~K}$. In particular, ab initio calculations allow a direct insight into the microscopic mechanisms determining the transport, and the acoustic-optical phonon interaction turns out to determine the magnitude of the lattice thermal conductivity in bismuth, thus allowing us to explain the discrepancies between previous theoretical work [28] and experiments [26]. Moreover, we show that the available experimental data for the LTC for polycrystalline thin films are remarkably explained by our calculations, which enables us to predict the effect of LTC size reduction for various temperatures and nanostructure shapes and sizes.

More precisely, we have first validated our formalism for the calculation of bulk bismuth in the binary direction by comparing with available experiments (Sec. III A). In a second step, we have validated our theoretical values with available experimental data for polycrystalline thin films (Sec. IV A). 
These validation steps have enabled us to predict in bulk bismuth both the lattice and electronic contributions in the trigonal direction. In the binary direction, they are predicted at temperatures at which only total conductivity measurements are available (Sec. III B). Then the heat transport reduction factor is given for thin films, nanowires and spherical grains, and in particular both for semiconducting and metallic films (Sec. IV B). Finally, the nanoscale mechanisms for the heat reduction in nanostructures have been analyzed in Sec. V.

\section{CALCULATION METHODS}

The effect of nanostructuring is studied by introducing a scattering term - the Casimir scattering rate $S_{v}$ - that enables us to account for the presence of grain boundaries and sample boundaries, by which phonons are scattered-in addition to phonon-phonon scattering. These scattering processes modify the out-of-equilibrium phonon distribution $N_{v}$ that enters the linearized Boltzmann transport equation [30-32].

\section{A. Nanostructuring}

The Casimir scattering rate for a phonon $v$ reads

$$
S_{v}=\frac{\left|\mathbf{c}_{v}^{b}\right|}{L^{\text {Cas } F}} N_{\nu}^{0}\left(N_{v}^{0}+1\right),
$$

where $L^{\text {Cas }}$ is the Casimir length and $F$ characterizes the sample roughness [32], taken to be 0.5 in all of our calculations $[30,31,33] . \quad N_{v}^{0}$ is the temperature-dependent equilibrium Bose-Einstein distribution of the phonon, $\left|\mathbf{c}_{v}^{b}\right|$ depends on the phonon group velocity $\mathbf{c}_{v}$ in the direction(s) in which the phonon transport is limited by the sample boundaries, and, thus, on the sample geometry. For instance, for spherical samples $\left|\mathbf{c}_{v}^{b}\right|=\left|\mathbf{c}_{v}\right|$, for nanowires $\left|\mathbf{c}_{v}^{b}\right|=\sqrt{\left(\mathbf{c}_{v}^{\text {cross }, 1}\right)^{2}+\left(\mathbf{c}_{v}^{\text {cross }, 2}\right)^{2}}$, where $c_{v}^{\text {cross, } i}$ are the phonon group velocity components perpendicular to the wire growth direction, and for thin films $\left|\mathbf{c}_{v}^{b}\right|=\left|\mathbf{c}_{v}^{\text {perp }}\right|$, where $\mathbf{c}_{v}^{\text {perp }}$ is phonon group velocity perpendicular to the thin-film plane (see also Appendix A for details).

The validation of Eq. (1) for polycrystalline thin films has been performed by taking into account the scattering by sample boundaries and by spherical grains inside the sample as two independent scattering rates:

$$
S_{v}=S_{v}^{\text {grain }}+S_{v}^{\text {film }} .
$$

Finally, the heat transport reduction factor has been predicted with $L^{\text {Cas }}$ taken to be either the thickness of singlecrystalline thin films or the nanoscopic dimension of spherical grains of polycrystalline samples.

\section{B. Lattice thermal conductivity}

The lattice thermal conductivity reads

$$
\kappa_{L}=\frac{\hbar^{2}}{k_{B} T^{2} V_{0}} \sum_{\nu} N_{v}^{0}\left(N_{v}^{0}+1\right) c_{v}^{\text {heat }} \omega_{v} \Delta N_{v},
$$

where the key ingredient, $\Delta N_{v}=N_{v}-N_{v}^{0}$, is the deviation of the phonon population with respect to $N_{v}^{0}$ under the influence of the phonon-phonon and phonon-boundary $\left(S_{v}\right)$ scattering terms. The quantity $c_{v}^{\text {heat }}$ is the projection of the phonon group velocity on the heat flux direction, the phonon frequency is $\omega_{v}$, $k_{B}$ is the Boltzmann constant, and $V_{0}$ is the unit-cell volume.

As it is well known, the amount of heat carried by different phonons depends on the phonon mean free paths which, in turn, depend on the phonon group velocities and anharmonic phonon lifetimes. To calculate the latter, the two main ingredients are the third-order anharmonic constants and the joint density of states (JDOS). For an initial incoming phonon $|\nu\rangle=|\mathbf{q}, \lambda\rangle$ of wave vector $\mathbf{q}$ and mode index $\lambda$, JDOS reads

$$
\begin{aligned}
J_{\mathbf{q} \lambda}= & \sum_{\mathbf{q}^{\prime} \lambda^{\prime}, \mathbf{q}^{\prime \prime} \lambda^{\prime \prime}} \delta\left(\hbar \omega_{\mathbf{q} \lambda}+\hbar \omega_{\mathbf{q}^{\prime} \lambda^{\prime}}-\hbar \omega_{\mathbf{q}^{\prime \prime} \lambda^{\prime \prime}}\right) \\
& +\delta\left(\hbar \omega_{\mathbf{q} \lambda}-\hbar \omega_{\mathbf{q}^{\prime} \lambda^{\prime}}-\hbar \omega_{\mathbf{q}^{\prime \prime} \lambda^{\prime \prime}}\right) .
\end{aligned}
$$

Here, the first $\delta$ function corresponds to a coalescence process, in which $|\mathbf{q}, \lambda\rangle$ and $\left|\mathbf{q}^{\prime}, \lambda^{\prime}\right\rangle$ phonons interact to create a final $\left|\mathbf{q}^{\prime \prime}, \lambda^{\prime \prime}\right\rangle$ phonon, and the second $\delta$ function describes the decay of a $|\mathbf{q}, \lambda\rangle$ phonon, accompanied by the creation of two phonons $\left|\mathbf{q}^{\prime}, \lambda^{\prime}\right\rangle$ and $\left|\mathbf{q}^{\prime \prime}, \lambda^{\prime \prime}\right\rangle$. One can then introduce a JDOS which depends on the frequency of the initial phonon, or $\omega$-dependent JDOS:

$$
J(\omega)=\sum_{\mathbf{q} \lambda} J_{\mathbf{q} \lambda} \delta\left(\omega_{\mathbf{q} \lambda}-\omega\right) .
$$

In our work, phonon frequencies and group velocities have been computed within the density functional perturbation theory (DFPT) [36] on a $6 \times 6 \times 6$ q-point grid in the Brillouin zone (BZ) centered [37] at $\Gamma$. The frequency gap between the highest acoustic branch and the lowest optical branch turns out to be of crucial importance in the calculation of the lattice conductivity (see Sec. III A), and an (upward) rigid shift of $12.5 \mathrm{~cm}^{-1}$ has been imposed on the frequencies of the optical phonon branches. State-of-the-art calculations of the third-order anharmonic constants have been performed on a $4 \times 4 \times 4$ q-point grid in the BZ and interpolated on denser grids [38]. In BTE, the LTC was accurately converged on a $28 \times 28 \times 28$ q-point grid in the BZ with a Gaussian broadening of the detailed balance condition [30] taken to be $\sigma=1 \mathrm{~cm}^{-1}$. Other technical details are reported in Appendix B.

\section{THERMAL TRANSPORT IN BULK BI}

\section{A. Lattice thermal conductivity}

The magnitude of the LTC in bismuth [Fig. 1, panel (a)] turns out to be controlled by the phonon joint density of states [JDOS, Eq. (4) and Eq. (5)] available for the coalescence of two acoustic phonons into an optical one (Fig. 2, arrow in the right panel). In turn, the JDOS around $40 \mathrm{~cm}^{-1}$ is extremely sensitive to the frequency of the lowest optical phonon branch near zone center. When the latter corresponds to the experimental one at $\Gamma$, i.e., $73 \mathrm{~cm}^{-1}$ at $\Gamma$ (Fig. 2, left panel, solid line and green circles), the JDOS is much smaller and, consequently, the calculated lattice thermal conductivity is higher and agrees with the available experimental values for the LTC [26] [Fig. 1, panel (a), solid line and green symbols]. When the average optical frequency is lower with respect to experiment as in the ab initio calculation (Fig. 2, 

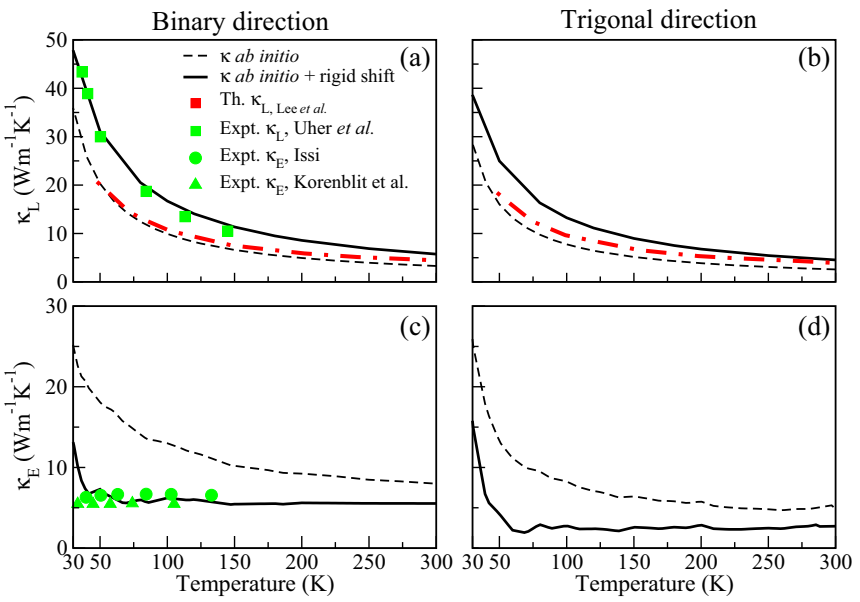

FIG. 1. Bulk bismuth. Lattice [panels (a) and (b)] and electronic [panels (c) and (d)] thermal conductivity (TC) as functions of temperature. Left panels: binary direction. Right panels: trigonal direction. Solid lines: calculation with experimentally observed AOPI. Dashed lines: $a b$ initio calculations. Green symbols: TC from expt. of Refs. [1,26,34]. Red dash-dotted line: previous ab initio calculation from Ref. [28]. Our electronic contribution was obtained as a difference between the experimentally observed total TC of Ref. [26] [panel (c)] or Ref. [35] [panel (d)] and our calculated LTC.

dashed line and green circles), the coalescence channel is enlarged for heat-carrying acoustic phonons, by emission of an optical phonon, and in this case our values of the LTC are smaller [Fig. 1, panel (a), dashed line] and similar to the values calculated in a previous ab initio calculation [28] (red dash-dotted line).

The acoustic-optical phonon interaction (AOPI) thus controls the heat transport by acoustic phonons in bismuth, and the AOPI turns out to itself be governed by the energy conservation rule near zone center present in Eq. (4). A correct description

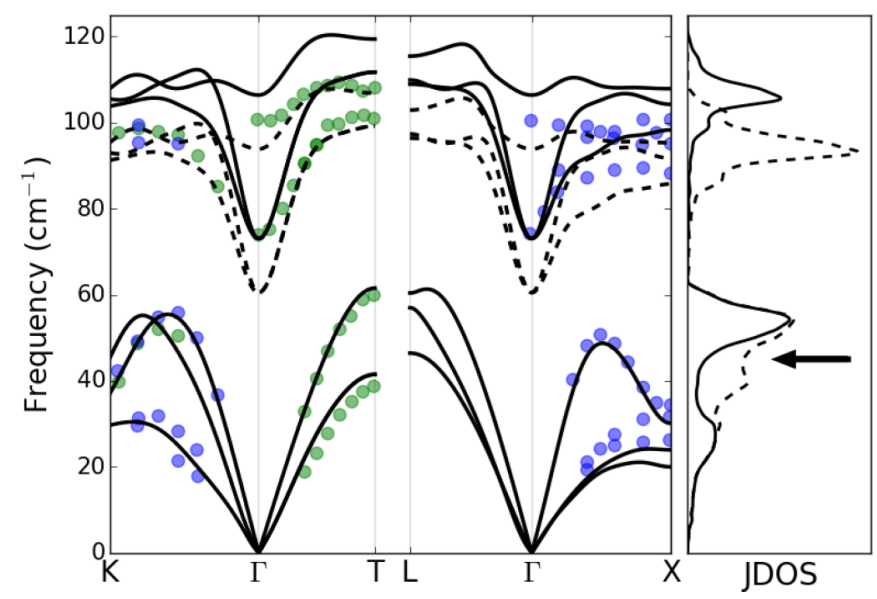

FIG. 2. Bulk bismuth. Left panel: phonon frequencies along some directions of high symmetry in the BZ. Solid line: the theoretical dispersion used in this work. Dashed line: optical phonon branches obtained with the $a b$ initio calculation. Right panel: joint density of states (JDOS) available for the phonon-phonon interaction. The JDOS for the acoustic-optical phonon interaction (AOPI) is marked with an arrow. Green and blue circles: expt. from Refs. [39,40]. of the energy threshold at which the coalescence of two acoustic phonons into an optical one occurs is obtained with the rigid shift of the optical branches. Contrastingly, a precise description of the optical branch over the whole BZ is less important as the heat carried by optical phonons represents only about $16.5 \%$ of the LTC at ambient temperature and becomes negligible at lower temperatures, as we will show in Sec. V of this work.

As can be seen in panels (a) and (b) of Fig. 1, the experimental values for the LTC are available only in the binary direction and in the restricted 30-150 K temperature range. As already mentioned in the Introduction, in semimetallic bulk bismuth electrons and phonons contribute to the TC at the same time [1]. Experimentally, separating these two contributions is quite a challenging task. Uher et al. [26] were able to measure the contribution to TC due to the charge carriers in the binary direction up to $T \approx 150 \mathrm{~K}$ by applying a very strong magnetic field, the method being limited to relatively low temperatures [26]. Alternatively, Gallo et al. [27] evaluated the electronic part by applying WiedmannFranz law and by using an analytical expression for the electronic contribution to the TC, the latter method being highly unreliable due to the limited precision of the models used [28]. In both Refs. [26] and [27], the lattice contribution has been found as the difference between the measured total thermal conductivity and the obtained electronic thermal conductivity.

At ambient temperature, an experimental data is no longer available in the binary direction. We find $\kappa_{L}=5.9 \mathrm{~W}(\mathrm{~K} \mathrm{~m})^{-1}$, a value higher by $30 \%$ than the calculation of Lee et al. [28], and widely different from the result of Ref. [27].

Then, turning to the trigonal direction, no reliable experimental data for the LTC as a function of temperature are available, to the best of our knowledge, for the reasons explained above. Our predicted LTC for the trigonal direction as a function of temperature in shown in Fig. 1, panel (b). At $300 \mathrm{~K}$, our predicted LTC in the trigonal direction is $4.5 \mathrm{~W}(\mathrm{~K} \mathrm{~m})^{-1}$, very close to the one of Ref. [28]. Thus we confirm the inaccuracy of the estimation for the bulk LTC made in Ref. [27], already pointed out in Ref. [28].

Our calculated LTC in the binary direction turns out to be by about one-third larger than in the trigonal direction, and we find the anisotropy ratio of the LTC $\frac{\kappa_{\perp}}{\kappa_{\|}}$to be quasiconstant and equal to 1.3 over a large temperature range, from $30 \mathrm{~K}$ to $500 \mathrm{~K}$.

In conclusion, our calculations of the LTC in bulk bismuth have been validated by comparison with available experimental data in the binary direction. The lattice thermal conductivity has been predicted in bulk bismuth in the trigonal direction by our calculations, and its anisotropy determined as a function of the temperature. Our LTC calculation can then be used to obtain the electronic contribution, as shown in the next paragraph.

\section{B. Electronic contribution}

The total thermal conductivity (TTC) of semimetallic Bi contains both lattice and electronic contributions. As already explained above, experimental data for the lattice thermal conductivity of $\mathrm{Bi}$ are quite scarce. On the contrary, experimental data for the total thermal conductivity are available in 
a wealth of experimental works [1,26,27,34,35]. We can use our calculated LTC to extract the electronic contribution from the TTC measurements $[26,35]$.

In panel (c) of Fig. 1, the available experimental data for the electronic part of the $\mathrm{TC}$ of $\mathrm{Bi}$ are shown by green symbols. In order to compare the results of our calculations with the data shown in panel (c) of Fig. 1, we have used the experimental data of Ref. [26] for the TC, from which we have extracted our calculated LTC with experimentally observed AOPI. As one can see, the agreement between our results and available experimental data shown in panel (c) of Fig. 1 is very good, as a constant value of $6 \mathrm{~W}(\mathrm{~K} \mathrm{~m})^{-1}$ for the electronic contribution to the TC is obtained in both theory and experiment in the binary direction of $\mathrm{Bi}$. Moreover, our calculations predict that the electronic contribution will stay constant and equal to $6.0 \mathrm{~W}(\mathrm{~K} \mathrm{~m})^{-1}$ over the whole $30-300 \mathrm{~K}$ temperature range. The constant behavior of the electronic contribution to $\mathrm{TC}$ as a function of temperature has been also observed experimentally; however, the reasons for this behavior, discussed for example in Ref. [26], are not trivial.

In the trigonal direction, the experimental results for the electronic contribution to the TC are not available. Our theoretical result shown in panel (d) of Fig. 1 has been obtained from the result for TTC of Ref. [35] and our calculated LTC with experimentally observed AOPI. As one can see, we predict a value of $3.0 \mathrm{~W}(\mathrm{~K} \mathrm{~m})^{-1}$ for the electronic contribution to the $\mathrm{TC}$ in the trigonal direction of $\mathrm{Bi}$.

For the sake of comparison, the electronic contributions obtained from measured TTC $[26,35]$ and $a b$ initio LTC without shift of optical phonon branches are also shown in panels (c) and (d) of Fig. 1. For these results shown in dashed lines, not only the predicted value for the electronic contribution to the TC differs from the experiments in the binary direction, but, much more importantly, the experimentally observed constant behavior of the electronic contribution to the TC as a function of temperature is not reproduced in the case of overestimated AOPI.

In conclusion of this paragraph, our calculations predict constant values of $6.0 \mathrm{~W}(\mathrm{~K} \mathrm{~m})^{-1}$ and $3.0 \mathrm{~W}(\mathrm{~K} \mathrm{~m})^{-1}$ for the electronic contribution to the $\mathrm{TC}$ in $\mathrm{Bi}$, in binary and trigonal directions, in the 30-300 K temperature range. This prediction is supported by the available experimental data, and thus can be further used to supplement the lack of experimental data, and to test future future approaches to compute the electronic contribution. In the present work, we will use it to predict the reduction in the total thermal conductivity in metallic nanostructures (see Sec. IVC3).

\section{THERMAL TRANSPORT IN NANOSTRUCTURED BI}

It is important to stress that the nanostructures whose size is a few tens of nanometers are on the verge of the confinement-induced semimetal-to-semiconductor transition [41]. Data about this transition are still scarce and depend on the nanostructure shape [42] and sample preparation conditions. This is the reason why, in the following, we study both poly- and monocrystalline nanostructures, as well as semiconducting and conducting films.

Before discussing the nanostructures, we note that describing the sample boundaries though Casimir's scattering rate, as we have done here, yields very different results with respect to the effect of size reduction on the LTC, compared to the accumulated approach, which is a typical approach used in the literature $[43,44]$. The comparison between the two approaches is shown in Appendix C.

\section{A. Polycrystalline samples}

Turning to the comparison of our calculated LTC with available experiments for Bi polycrystalline thin films [18], we note that, in polycrystalline samples, the nanostructuring effect is determined both by the film thickness, and by the average grain size [18]. In the work of Ref. [18], the average grain size was measured for every thin film investigated. While for very thin films, the average grain size was found to be larger than the film thickness, for films thicknesses larger than $200 \mathrm{~nm}$, the average grain size was inferior to the film thickness (Fig. 3 in Ref. [18]). In our calculation, we took into account both scattering processes through Eq. (2), using the experimental grain sizes of Ref. [18] (Fig. 3, solid lines). As one can see in Fig. 3, we find a remarkable agreement with the results of Ref. [18] (red diamonds). The fact that the theoretical LTCs are close to the experimental total thermal conductivities is consistent with the marked semiconducting temperature-dependent behavior seen in the film electrical resistivity [18].

In Fig. 3, the available experimental data of Ref. [19] is also shown (empty squares). In Ref. [19], the average grain size was found to be approximately equal to the film thickness, i.e., it was found to be larger than that of Ref. [18] for
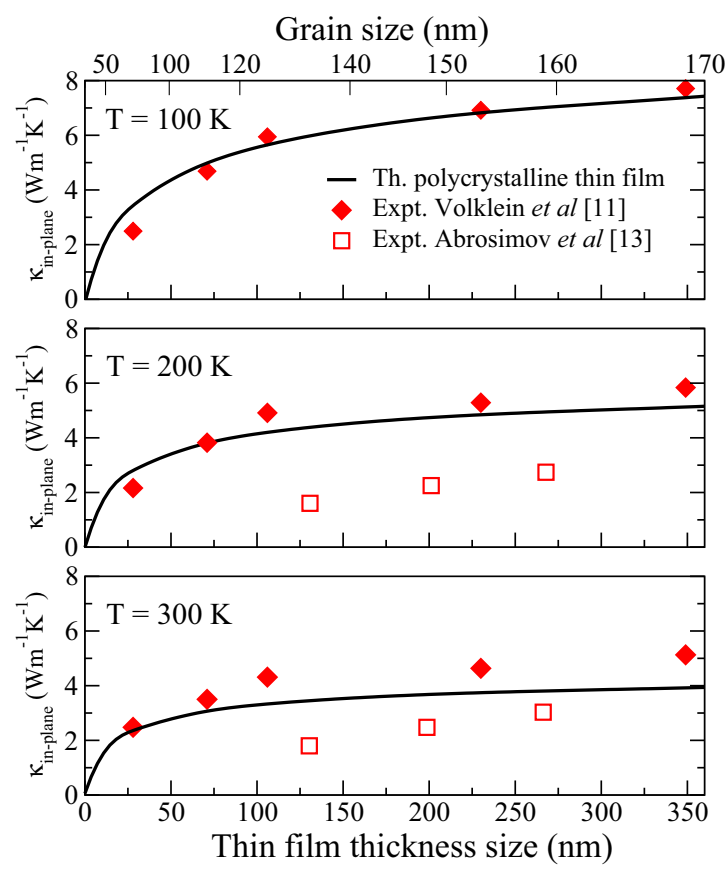

FIG. 3. Bi thin films. Lattice thermal conductivity in the binary direction (solid lines) as a function of the thin-film thickness (nm) (bottom abscissa axis) or of the grain size (top abscissa axis; data from Ref. [18]) at $100 \mathrm{~K}, 200 \mathrm{~K}$, and $300 \mathrm{~K}$ (top, center, and bottom panels). Red filled diamonds: expt. total thermal conductivity $\kappa_{\text {TOT }}$ from Ref. [18]. Empty red squares: expt. $\kappa_{\text {Tот }}$ from Ref. [19]. 

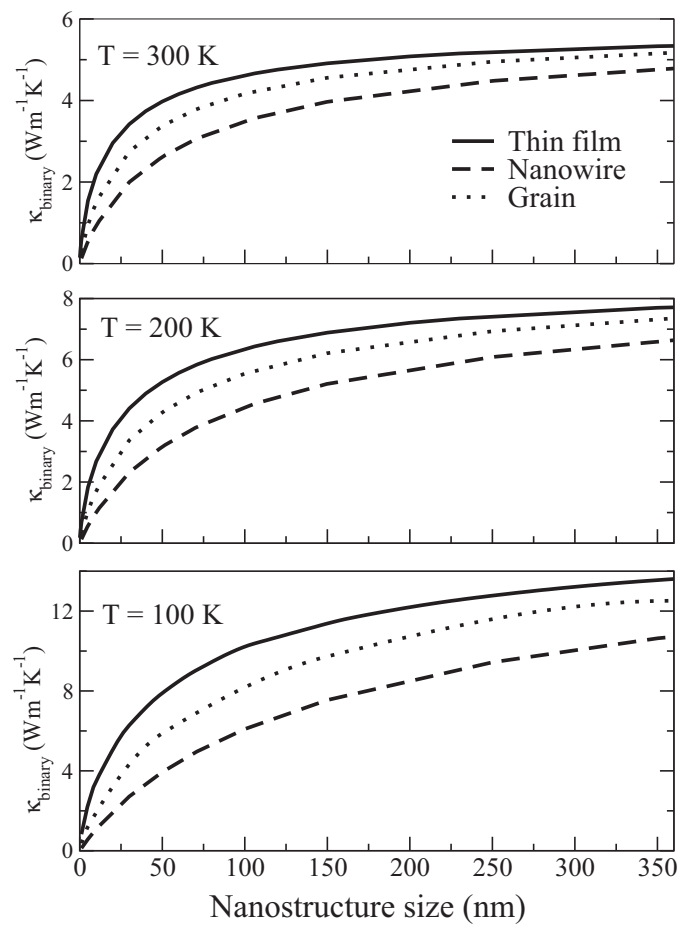

FIG. 4. Lattice thermal conductivity (LTC) for different geometries of bismuth nanostructures. Solid lines: LTC in the binary direction for monocrystalline thin films as a function of the film thickness. Dotted lines: LTC along the binary axis of monocrystalline nanowires. Dashed lines: spherical grain.

most samples. Although our calculated LTC lies between the two experimental data sets for $200 \mathrm{~K}$ and $300 \mathrm{~K}$, the best agreement is clearly found for the more recent work [18]. The most probable cause of discrepancy between the results of Refs. [18] and [19] is the effect of the film substrate in the latter work, as pointed out in Ref. [18].

\section{B. Monocrystalline samples}

The effect of size reduction on the LTC for monocrystalline samples of different geometries is shown in Fig. 4. For Bi nanowires, our theory shows, in agreement with experiments, a monotonical decrease of the LTC when the diameter decreases. But in contrast to polycrystalline thin films, the experimental data for $\mathrm{Bi}$ nanowires available in literature are extremely scattered [2,21-25], suggesting the presence of unidentified sample-dependent scattering mechanisms in Bi [2] as well as in $\mathrm{Si}$ [14-16]. Available theoretical studies suggest a profound modification of the nanowire atomic structure such as surface oxidation, roughness, and core defects [14-16], which are beyond the scope of this work.

\section{Heat transport reduction}

\section{Semiconducting nanostructures}

Having shown the predictive capability of our method for polycrystalline films, we discuss the reduction of thermal transport, which has been rationalized with the concept of the reduction factor which we define as $R(T)=\frac{\kappa_{L}^{\text {bulk }}(T)}{\kappa_{L}^{\text {nano }}(T)}$ for

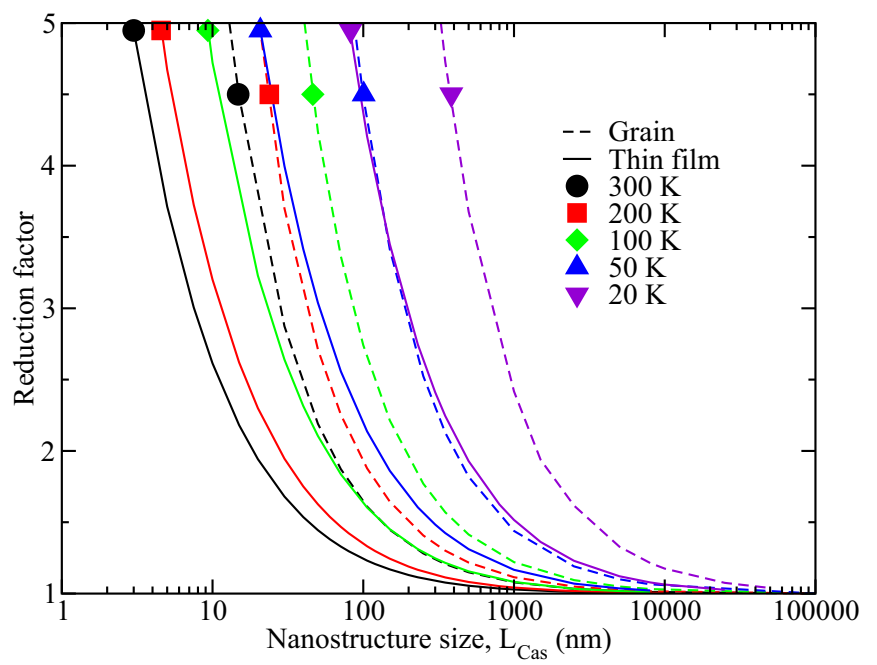

FIG. 5. Nanostructuring-reduced lattice thermal conductivity (LTC). Abacus of the reduction factor as a function of the nanostructure size and temperature, at $300 \mathrm{~K}$ (black), $200 \mathrm{~K}$ (red), $100 \mathrm{~K}$ (green), $50 \mathrm{~K}$ (blue), and $20 \mathrm{~K}$ (violet). Solid lines: single-crystalline thin films, LTC along the binary direction; dashed lines: spherical grain geometry.

semiconducting nanostructures (Fig. 5). As already demonstrated in Fig. 4, spherical shape (dashed line) turns out to yield a calculated reduction factor higher than the geometry of a single-crystalline film (solid lines) or a single-crystalline wire. Spherical grains are most commonly found in polycrystalline samples and here we assume, in constrast to Fig. 3, that the sample size is significantly larger than the grain size. In fact, in cases where only thermal properties matter and electronic conductivity is not so important, the control of grain sizes in polycrystalline films turns out to provide the best strategy for the control of heat transport reduction in bismuth. For instance, for a target reduction factor equal to two, the grain size should be smaller than $62 \mathrm{~nm}$ at $300 \mathrm{~K}, 95 \mathrm{~nm}$ at $200 \mathrm{~K}$, and $188 \mathrm{~nm}$ at $100 \mathrm{~K}$ (dashed lines). Figure 5 thus provides an abacus for the thermal management that can be employed to deduce the reduction factor for a given nanostructure size or, alternatively, to choose the nanostructure size to obtain a desired reduction of the heat transport.

\section{Semiconducting nanofilms}

But in applications for thermoelectricity, it is important to keep the electrical conductivity as high as possible. In this case, the use of polycrystalline samples is precluded because the electronic scattering by grain boundaries lowers the electrical conductivity, for instance by a factor of five at ambient temperature in polycrystalline thin films with respect to monocrystalline ones [18]. Electrical contacts are also very important, and the fabrication of contacts is facilitated in the thin-film geometry. Therefore, we show the reduction factor for single-crystalline thin films (Fig. 5, solid lines). The reduction factor turns out to be lower than for spherical grains, which can be compensated by decreasing the film thickness by about a factor of three. For a target reduction factor of two, the film 


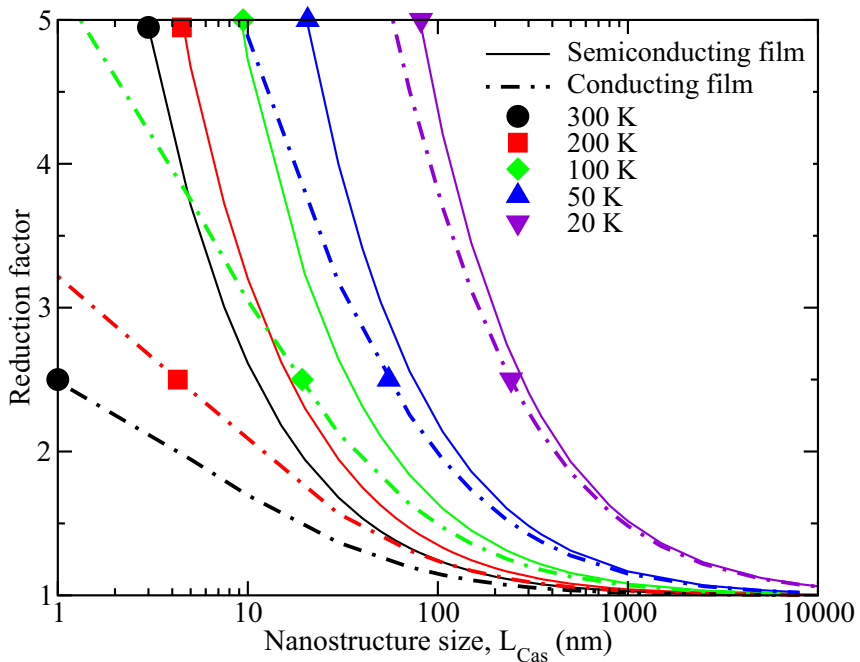

FIG. 6. Nanostructuring-reduced total thermal conductivity in the binary direction of single-crystalline thin films. Solid lines: semiconducting thin films (same as Fig. 5). Dash-dotted lines: conducting thin films. Same color legend as in Fig. 5.

thickness should be smaller than $19 \mathrm{~nm}$ at $300 \mathrm{~K}, 27 \mathrm{~nm}$ at $200 \mathrm{~K}$, and $60 \mathrm{~nm}$ at $100 \mathrm{~K}$ (solid lines).

\section{Conducting nanofilms}

Recently, fabrication of very high quality Bi films became possible [41] in which the electrical conductivity is kept to the semimetallic bulk value even for a thickness as small as $76 \mathrm{~nm}$. The reduction factor of the total thermal conductivity of semimetallic thin films has been estimated as a function of nanostructure size and temperature (dashed dotted curves) (Fig. 6). To this end, we have redefined the reduction factor as $\hat{R}(T)=\frac{\kappa_{L}^{\text {bulk }}+\kappa_{E}^{\text {bulk }}}{\kappa_{L}^{\text {nano }}+\kappa_{E}^{\text {bulk }}}$, and set the electronic contribution $\kappa_{E}$ to zero for a semiconducting nanostructure, while approximating it in the semimetallic nanostructure by the value for the bulk calculated in Sec. III B, $\kappa_{E}^{\text {bulk }}=6 \mathrm{~W}(\mathrm{~K} \mathrm{~m})^{-1}$ in the binary direction. This is reasonable for films in which the electrical conductivity is close to the bulk one [41].

The reduction of the total thermal conductivity is smaller in the case of metallic thin films (dashed dotted curves) than in the case of semiconducting ones (solid lines). Nonetheless, for a thickness of $76 \mathrm{~nm}$, the reduction factor amounts to 1.2 at $300 \mathrm{~K}, 1.3$ at $200 \mathrm{~K}$, and 1.6 at $100 \mathrm{~K}$. Thus an experimental investigation of $Z T$ in semimetallic single-crystalline thin films would be of some interest for thermoelectric applications.

\section{ANALYSIS OF HEAT TRANSPORT MECHANISMS}

In this section, we analyze the contributions to the LTC of bulk and nanostructured bismuth from different phonon modes, as well as the distribution over the Brillouin zone.

\section{A. Roles of different phonons}

In Fig. 7 we show relative contributions to the vibrational part of the thermal conductivity of bulk bismuth (thin lines), due to different acoustic phonon modes, as well as the one from the optical modes, as functions of temperature.

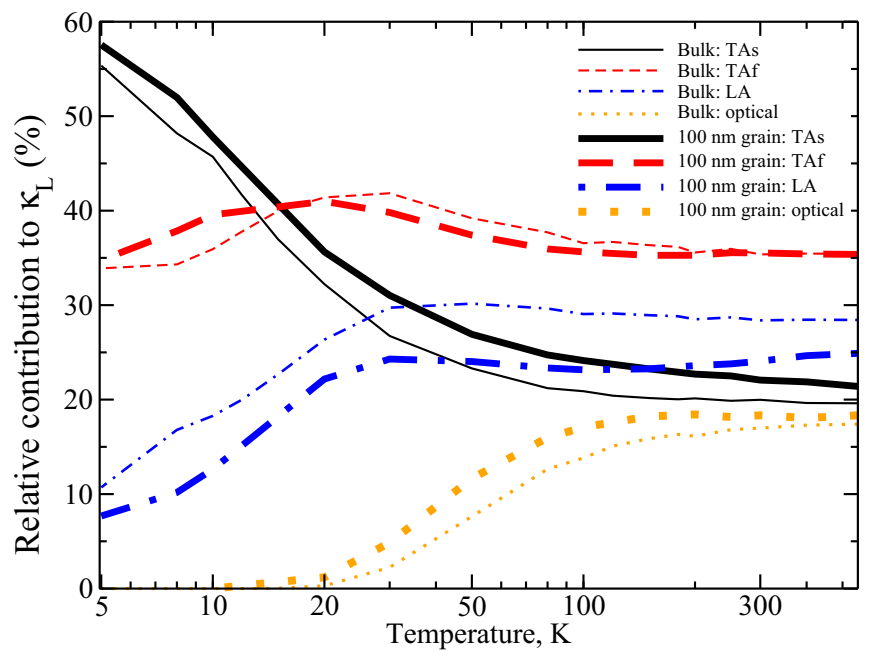

FIG. 7. Bulk (thin lines) and nanostructured (thick lines) Bi: relative contributions of different phonon modes to the lattice thermal conductivity in the binary direction. Nanostructured Bi with $100 \mathrm{~nm}$ grain geometry. Solid lines: TAs phonons. Dashed lines: TAf phonons. Dash-dotted lines: LA phonons. Dotted lines: optical phonons. We define TAf phonons, where "f" stands for fast, as those having a larger sound velocity than the one of TAs phonons, where "s" stands for slow.

As one can see in Fig. 7, at ambient temperature, the major contributions to the LTC come from energetic longitudinal acoustic (LA) and fast transverse acoustic (TAf) phonons. While the relative importance of the highest acoustic modes stays almost unchanged in the 30-300 K temperature range, the contribution of optical modes, which represents $16.5 \%$ at ambient temperature, decreases rapidly with temperature and becomes zero at $20 \mathrm{~K}$. The decrease of the role of optical phonons is accompanied by the increase of the role of the lowest acoustic (TAs) mode. As the temperature becomes lower than $30 \mathrm{~K}$, the relative contribution of the most energetic acoustic modes starts to decrease also, due to the decreasing population of phonon states, while the role of TAs increases even faster. At low temperatures, the two lowest acoustic branches carry about $90 \%$ of heat, and the dominant contribution comes from the lowest transverse acoustic branch TAs $(55 \%)$. The behavior of the relative contributions of the acoustic phonons in the trigonal direction as a function of temperature (not shown) was found very similar to that in the binary direction.

Turning to the relative roles of different phonons in the LTC of nanostructured Bi (results for $100 \mathrm{~nm}$ spherical grains shown in Fig. 7 in thick lines), the most significant effect with respect to bulk is the reduction of the role of LA phonon in nanostructures (dash-dotted lines). This is not surprising, as LA phonons have the highest group velocities and the longest mean free paths, which are expected to be the most affected by the nanostructure boundaries.

\section{B. Distribution over the Brillouin zone}

In Fig. 8, we have analyzed our calculated LTC $\kappa_{L}$ of Eq. (3) as a function of the modulus of the wave vector of the initial phonon $|\mathbf{q}|$, for bulk Bi and several nanostructure sizes in 


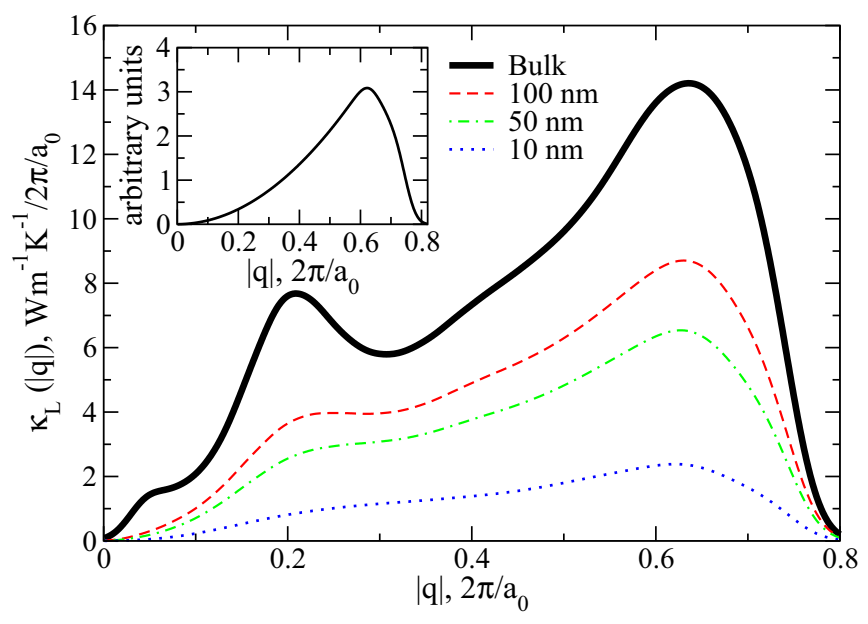

FIG. 8. Bulk (thick line) and nanostructured bismuth (thin lines): Brillouin zone distribution of the LTC as a function of $|\mathbf{q}|$ of the initial phonon, in the binary direction and at $300 \mathrm{~K}$. Nanostructured bismuth in the grain geometry of diameter $100 \mathrm{~nm}$ (dashed line), $50 \mathrm{~nm}$ (dash-dotted line), and $10 \mathrm{~nm}$ (dotted line). Inset panel: the density of q-phonon states.

the grain geometry, at $300 \mathrm{~K}$. We introduce $\kappa_{L}(|\mathbf{q}|)$, which is defined by the following relation: $\kappa_{L}=\int \kappa_{L}(|\mathbf{q}|) d|\mathbf{q}|$. Only the LTC in the binary direction is presented in Fig. 8, while a detailed analysis is reported in Appendix C.

As can be seen in Fig. 8, at $300 \mathrm{~K}$, the magnitude of the LTC both in bulk and nanostructures is due to phonons with $|\mathbf{q}|$ vectors all over the Brillouin zone, and not only to zone-center phonons. In the case of the bulk material (thick solid line), this is somewhat surprising, as one expects the main contribution to the LTC to come from the zone center, as the region around the $\Gamma$ point of the $\mathrm{BZ}$ corresponds to the smallest broadenings and to the highest group velocities and thus to the highest phonon mean free paths. One can indeed see in Fig. 8 a peak in the bulk LTC around $|\mathbf{q}|=0.22 \pi / a_{0}$, corresponding to LA phonons with large mean free paths (see Appendix C), but this peak does not represent the dominant contribution to the LTC.

This relatively uniform behavior of the contributions of phonons with different $|\mathbf{q}|$ vectors can be understood, if one remembers that the quantity $\kappa_{L}$ contains, implicitly, the density of states of the initial phonon. It must be noted that the phonon DOS, calculated as a function of $|\mathbf{q}|$, depends only on the form of the $\mathrm{BZ}$, and, in the case of $\mathrm{Bi}$, shows parabolic dependence on $|\mathbf{q}|$ except at the zone boundaries. Indeed, away from the boundaries, the $\mathrm{BZ}$ of $\mathrm{Bi}$ is almost "spherical." Thus we expect to find most of the phonon states at large $|\mathbf{q}|$ close to BZ boundaries. The phonon DOS is shown in the inset of Fig. 8 . Indeed, at smallest $\mathbf{q}$ vectors, the density of phonon states is very small. Thus the region around $\Gamma$ where the group velocities are large and broadenings are small, resulting in large phonon mean free paths, gives a relatively small contribution to the thermal conductivity.

Turning to nanostructures (thin lines) one can see that the peak contribution of the LA phonons with large mean free paths is rapidly killed by boundary scattering (already at $100 \mathrm{~nm}$ ), while other contributions over the BZ are almost uniformly reduced with the reduced nanostructure size.

\section{CONCLUSION}

In conclusion, in this work, we have calculated the lattice thermal conductivity of bismuth in a large temperature range for bulk and for nanostructures, finding results in excellent agreement with available experiments. Acoustic-optical phonon interaction turns out to have a preeminent role in the magnitude of the LTC. Finally, we predict the effect of size reduction for various temperatures and nanostructure shapes, and show that the largest heat transport reduction is obtained in polycrystals with grain sizes smaller than $100 \mathrm{~nm}$. The reduction factor is also predicted for semimetallic single-crystalline thin films. We analyze the contribution from the different heat-carrying phonons and their distribution in the Brillouin zone, as a function of the temperature, showing that the contribution of LA phonons to LTC is the most affected by temperature and size reduction.

\section{ACKNOWLEDGMENTS}

We acknowledge fruitful discussions with A. McGaughey, B. Fauquet, and G. Kané. The QUANTUM ESPRESSO package [45] has been used in this work. We acknowledge support from the DGA. Computer time has been granted by GENCI (Project No. 2210) and by École Polytechnique through the LLR-LSI project.

\section{APPENDIX A: SAMPLE GEOMETRY DESCRIPTION}

In bulk bismuth, because of the uniaxial symmetry, the transport properties are determined by measuring LTC along the trigonal axis (trigonal direction denoted $\|$ ) and perpendicular to it (binary direction denoted $\perp$ ). The third axis which defines the plane perpendicular to the trigonal axis, additional to the binary direction, is called the bisectrix direction. In bulk bismuth, the binary and bisectrix directions are equivalent by symmetry, and the LTC values are the same in these two directions.

For the samples of finite size, the different sample geometries can be thin films, wires, and spherical nanoparticles (Fig. 9). For the sake of generality, in Fig. 9, we use Cartesian coordinates, and the heat flux is measured in the $y$ direction. If, for example, the heat flux is measured in the binary direction, then the $z$ axis will be the one which corresponds to the trigonal direction, and $x$ axis will denote the bisectrix direction.

In Eq. (1) of the main text, $\left|\mathbf{c}_{v}^{b}\right|$ is the phonon group velocity $\mathbf{c}_{v}$ in the direction in which the phonon transport is limited by the sample boundaries. It is thus different for different sample geometries and depends on the sample orientation in space with respect to the direction in which heat flux is measured.

For instance, in the case of configurations shown in Fig. 9, we have $\left|\mathbf{c}_{v}^{b}\right|=\left|c_{v}^{z}\right|$ for the thin film geometry, $\left|\mathbf{c}_{v}^{b}\right|=\sqrt{\left(c_{v}^{x}\right)^{2}+\left(c_{v}^{z}\right)^{2}}$ for the nanowire geometry, and $\left|\mathbf{c}_{v}^{b}\right|=$ $\sqrt{\left.\left(c_{\nu}^{x}\right)^{2}+\left(c_{\nu}^{y}\right)^{2}+\left(c_{\nu}^{z}\right)^{2}\right)}$ for the spherical-grain geometry. Here, $c_{v}^{x}, c_{v}^{y}, c_{v}^{z}$ are Cartesian components of the phonon group velocity $\mathbf{c}_{v}$. 


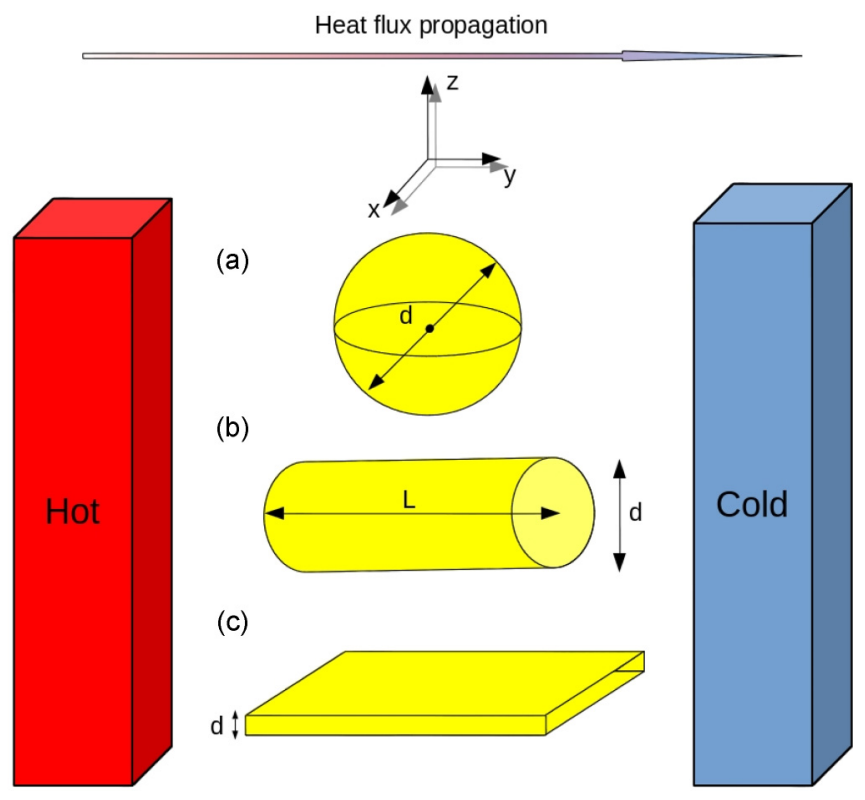

FIG. 9. Illustration of different sample geometries: spherical grain, wire, and thin film.

\section{APPENDIX B: TECHNICAL DETAILS}

A fully relativistic norm-conserving pseudopotential with $5 d$ electrons frozen in the atomic core and including nonlinear core correction has been designed in this work. Calculations were performed with a plane-wave energy cutoff of $90 \mathrm{Ry}$ and the local density approximation (LDA). Spin-orbit coupling (SOC) was accounted for in the calculation of the electronic structure and vibrational properties.

At theoretical equilibrium, the calculated lattice parameters are $a_{0}=4.667 \AA, \alpha=57.99^{\circ}$, and $u=0.236$ (in units of $a_{0}$ ), which are very close to the experimental values $a_{0}=4.724 \AA$, $\alpha=57.35^{\circ}$, and $u=0.234$ [46]. A $20 \times 20 \times 20$ MonkhorstPack k-points grid has been used to sample the Brillouin zone and a band structure in extremely satisfactory agreement with the DFT-LDA calculations of Ref. [47]—where the $5 d$ electrons were considered as semicore states and treated with the valence electrons - has been obtained.

\section{APPENDIX C: ACCUMULATED VS CASIMIR APPROACH}

As mentioned in the main text, in our work the change in the phonon distribution $\Delta N_{\nu}$ has been varied in the presence of the sample boundaries, and/or in the presence of the grains inside the polycrystalline sample, through Casimir's scattering rate. At variance, the typical approach used in the literature to study the effect of size reduction, the accumulated approach $[43,44]$, is an a posteriori analysis of the results obtained for the bulk material. This consists in keeping the $\Delta N_{v}$ constant in Eq. (3), and in canceling the phonon contribution into the LTC when the phonon mean-free path is larger than the nanostructure size $[43,44]$.

As shown in Table I, for the prediction of the nanostructure size that would yield a $50 \%$ reduction of the LTC, our results of the accumulated approach are found in close agreement with those of Ref. [28]. In contrast, we find that nanostructure
TABLE I. Bi: maximum value of the heat carrier mean-free path $\Lambda(\mathrm{nm})$ which provides a contribution of $50 \%$ to the bulk lattice thermal conductivity in the binary direction.

\begin{tabular}{|c|c|c|c|}
\hline \multirow{2}{*}{$\begin{array}{l}\text { Method } \\
T(\mathrm{~K})\end{array}$} & \multicolumn{2}{|c|}{ Accumulated approach } & \multirow{2}{*}{$\begin{array}{c}\text { Casimir's scatterin } \\
\text { This work }\end{array}$} \\
\hline & Previous work ${ }^{\mathrm{a}}$ & This work & \\
\hline 10 & & 3380 & 10400 \\
\hline 20 & & 526 & 1430 \\
\hline 50 & 120 & 148 & 410 \\
\hline 100 & 55 & 69 & 185 \\
\hline 200 & 26 & 34 & 95 \\
\hline 300 & 17 & 22 & 62 \\
\hline
\end{tabular}

${ }^{\mathrm{a}}$ Lee et al. from Ref. [28].

sizes obtained with the accumulated approach are about three times smaller than those predicted using Casimir's scattering rate (see Table I), at all temperatures.

\section{APPENDIX D: DISTRIBUTION OVER THE BRILLOUIN ZONE}

We have analyzed our calculated LTC $\kappa_{L}$ of Eq. (3) as a function of the modulus of the wave vector of the initial phonon $|\mathbf{q}|$ (see main text). It is presented in Fig. 10 for $T=300 \mathrm{~K}$ (top panels) and for $T=5 \mathrm{~K}$ (bottom panels) in the binary (left panels) and trigonal (right panels) directions. We have also extracted the contributions of the three acoustic branches (thin lines).

The behavior of the LTC (thick solid lines) at $300 \mathrm{~K}$ was discussed in the main text in the binary direction. Results in the trigonal direction are very similar.

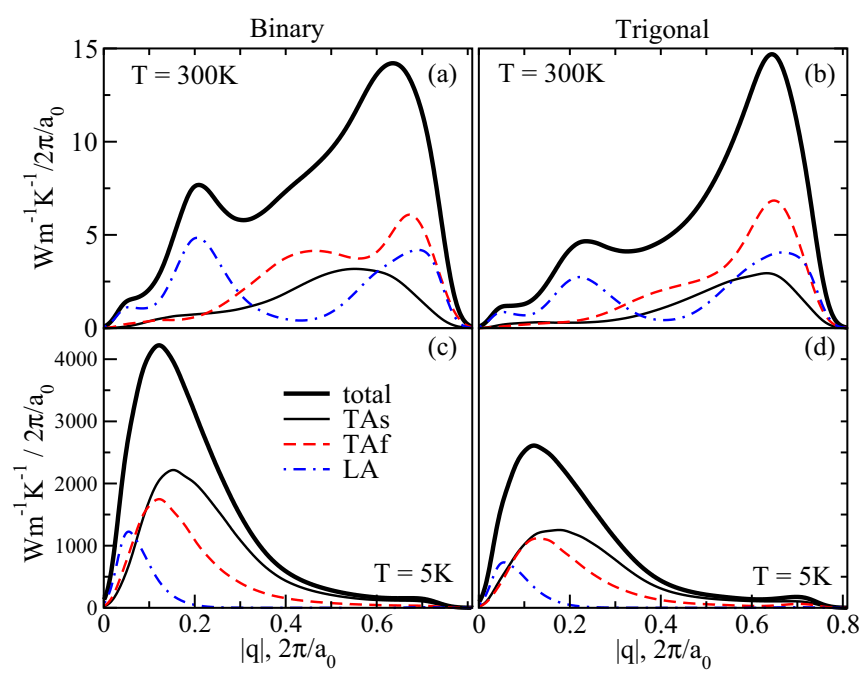

FIG. 10. Bulk Bi. Brillouin zone distribution of the acoustic phonon contributions (thin lines) to the LTC (thick lines) as a function of the modulus of the phonon vector of incoming phonon $|\mathbf{q}|$ in the binary (left) and trigonal (right) directions at $300 \mathrm{~K}$ (top) and $5 \mathrm{~K}$ (bottom). Thin solid lines: TAs branch. Thin dashed lines: TAf branch. Thin dash-dotted lines: LA branch. See caption of Fig. 7 for the definition of TAs and TAf. 
Coming to the contributions from separate phonon modes, as one can see in Fig. 10, at $|\mathbf{q}|$ smaller than $0.252 \pi / a_{0}$, the main contribution is due to the highest acoustic branch which has the highest group velocity and the smallest phonon-phonon linewidth. However, at $\mathbf{q}$ vectors larger than $0.252 \pi / a_{0}$ the phonon-phonon linewidth of the highest acoustic branch rapidly increases, and, in consequence, the contribution of this branch decreases. The contributions of the lowest acoustic branches, at $300 \mathrm{~K}$, show the behavior similar to that of the $|\mathbf{q}|$-dependent phonon DOS (inset panel of Fig. 8).

Contrastingly, at $T<20 \mathrm{~K}$ the major contribution to $\kappa_{L}(|\mathbf{q}|)$ starts to drift towards the small $|\mathbf{q}|$ since most of the high-energy phonon states are not populated due to the small thermal energy $k_{B} T$. The only active states are thus located near $\Gamma$. In the bottom panels of Fig. 10 we see the LTC $\kappa_{L}(|\mathbf{q}|)$ at $5 \mathrm{~K}$ which has a pronounced peak at $|\mathbf{q}| \approx 0.152 \pi / a_{0}$ for both directions.

By comparing right and left panels of Fig. 10, one can see that the contributions of all three phonon modes show anisotropy, at all values of $|\mathbf{q}|$, and at all temperatures. The anisotropy of the lattice thermal conductivity of bismuth, mentioned in the main text, is due at the same time to phonon group velocities, which differ in different directions, and to phonon lifetimes.
[1] J.-P. Issi, Aus. J. Phys. 32, 585 (1979).

[2] A. L. Moore, M. T. Pettes, F. Zhou, and L. Shi, J. Appl. Phys. 106, 034310 (2009).

[3] H. J. Goldsmid, Phys. Status Solidi A 1, 7 (1970).

[4] A. M. Ibrahim and D. A. Thompson, Mater. Chem. Phys. 12, 29 (1985).

[5] A. Popescu, L. M. Woods, and G. S. Nolas, Phys. Rev. B 85, 115202 (2012).

[6] L. D. Hicks and M. S. Dresselhaus, Phys. Rev. B 47, 16631 (1993).

[7] L. D. Hicks and M. S. Dresselhaus, Phys. Rev. B 47, 12727 (1993).

[8] J. P. Heremans, C. M. Thrush, D. T. Morelli, and M. C. Wu, Phys. Rev. Lett. 88, 216801 (2002).

[9] E. Shapira, A. Holtzman, D. Marchak, and Y. Selzer, Nano Lett. 12, 808 (2012).

[10] J. Szczech, J. Higgins, and S. Jin, J. Mater. Chem. 21, 4037 (2011).

[11] Q. Hao, G. Zhu, G. Joshi, X. Wang, A. Minnich, Z. Ren, and G. Chen, Appl. Phys. Lett. 97, 063109 (2010).

[12] C. Hua and A. J. Minnich, Semicond. Sci. Technol. 29, 124004 (2014).

[13] B. Liao, B. Qiu, J. Zhou, S. Huberman, K. Esfarjani, and G. Chen, Phys. Rev. Lett. 114, 115901 (2015).

[14] J. Carrete, L. J. Gallego, L. M. Varela, and N. Mingo, Phys. Rev. B 84, 075403 (2011).

[15] Y. He and G. Galli, Phys. Rev. Lett. 108, 215901 (2012).

[16] D. G. Cahill, P. V. Braun, G. Chen, D. R. Clarke, S. H. Fan, K. E. Goodson, P. Keblinski, W. P. King, G. D. Mahan, A. Majumdar et al., Appl. Phys. Rev. 1, 011305 (2014).

[17] J. Mendoza, K. Esfarjani, and G. Chen, J. Appl. Phys. 117, 174301 (2015).

[18] F. Völklein and E. Kessler, Phys. Status Solidi A 81, 585 (1984).

[19] G. Abrosimov, Teplofiz. Vys. Temp. 12, 530 (1974).

[20] D. W. Song, W.-N. Shen, B. Dunn, C. D. Moore, M. S. Goorsky, T. Radetic, R. Gronsky, and G. Chen, Appl. Phys. Lett. 84, 1883 (2004).

[21] J. P. Heremans, Acta. Phys. Pol. A 108, 609 (2005).

[22] J. W. Roh, K. Hippalgaonkar, J. H. Hal, R. Chen, M. Z. Li, P. Ercius, A. Majumdar, W. Kim, and W. Lee, ACS Nano 5, 3954 (2011).

[23] Y. Hasegawa, M. Murata, F. Tsunemi, Y. Saito, K. Shirota, T. Komine, C. Dames, and J. Garay, J. Electron. Mater. 42, 2048 (2013).
[24] S.-Y. Lee, G.-S. Kim, M.-R. Lee, H. Lim, W.-D. Kim, and S.-K. Lee, Nanotechnology 24, 185401 (2013).

[25] J. Kim, S. Lee, Y. M. Brovman, P. Kim, and W. Lee, Nanoscale 7, 5053 (2015).

[26] C. Uher and H. J. Goldsmid, Phys. Status Solidi B 65, 765 (1974).

[27] C. F. Gallo, B. S. Chandrasekhar, and P. H. Sutter, J. Appl. Phys. 34, 144 (1963).

[28] S. Lee, K. Esfarjani, J. Mendoza, M. S. Dresselhaus, and G. Chen, Phys. Rev. B 89, 085206 (2014).

[29] L. Paulatto, F. Mauri, and M. Lazzeri, Phys. Rev. B 87, 214303 (2013).

[30] G. Fugallo, M. Lazzeri, L. Paulatto, and F. Mauri, Phys. Rev. B 88, 045430 (2013).

[31] A. Sparavigna, Phys. Rev. B 65, 064305 (2002).

[32] J. M. Ziman, Electrons and Phonons: The Theory of Transport Phenomena in Solids (Oxford University Press, Oxford, 1960).

[33] D. A. Broido, L. Lindsay, and A. Ward, Phys. Rev. B 86, 115203 (2012).

[34] I. Y. Korenblit, M. E. Kuznetsov, V. M. Muzhdaba, and S. S. Shalyt, Sov. Phys. JETP 30, 1009 (1970).

[35] A. Collaudin, Ph.D. thesis, Université Pierre et Marie Curie Paris VI, France, 2014.

[36] S. Baroni, S. de Gironcoli, A. D. Corso, and P. Giannozzi, Rev. Mod. Phys. 73, 515 (2001).

[37] Spin-orbit coupling (SOC) was neglected in the calculation of the third-order anharmonic constants. Comparison with Ref. [28] shows that the effect of SOC is weak.

[38] The $4 \times 4 \times 4$ grid amounts to 95 irreducible $\left(\mathbf{q}_{1}, \mathbf{q}_{2}, \mathbf{q}_{3}\right)$ phonon triplets [29], where $\mathbf{q}_{i}, i=1,3$ are phonon wave vectors, and with $\mathbf{q}_{1}=\mathbf{q}_{2} \pm \mathbf{q}_{3}+\mathbf{G}$. $\mathbf{G}$ is a vector of the reciprocal lattice, and both normal and Umklapp processes have been taken into account exactly in the Boltzmann equation. The thirdorder anharmonic constants were Fourier-interpolated on the $28 \times 28 \times 28$ denser grid necessary for converged integrations in Eq. (3). The convergence has been checked at $T=5 \mathrm{~K}$ and $T=300 \mathrm{~K}$.

[39] J. L. Yarnell, J. L. Warren, R. G. Wenzel, and S. H. Koenig, IBM J. Res. Dev. 8, 234 (1964).

[40] D. B. Smith, Los Alamos Report No. 3773, 1967 (unpublished).

[41] M. Rudolph and J. J. Heremans, Appl. Phys. Lett. 100, 241601 (2012).

[42] A transition from a metallic to a semiconducting state has been found for diameters smaller than about $d=50 \mathrm{~nm}$ in $\mathrm{Bi}$ nanowires $[48,49]$ and of less than about $d=40 \mathrm{~nm}$ for bismuth 
nanotubes [50]. For films, a semiconducting (111) film with a metallic surface has been reported for a thickness of $90 \mathrm{~nm}$. A semimetallic Bi film has been synthetized [41] with a thickness as small as $76 \mathrm{~nm}$.

[43] C. Dames and G. Chen, Handbook of Thermoelectrics: Macro to Nano, edited by D. M. Rowe (CRC Press, Boca Raton, FL, 2006).

[44] A. S. Henry and G. Chen, J. Comput. Theor. Nanosci. 5, 141 (2008).

[45] P. Giannozzi, S. Baroni, N. Bonini, M. Calandra, R. Car, C. Cavazzoni, D. Ceresoli, G. Chiarotti, M. Cococcioni,
I. Dabo et al., J. Phys.: Condens. Matter 21, 395502 (2009).

[46] D. Schiferl and C. Barrett, J. Appl. Crystallogr. 2, 30 (1969).

[47] I. Timrov, T. Kampfrath, J. Faure, N. Vast, C. R. Ast, C. Frischkorn, M. Wolf, P. Gava, and L. Perfetti, Phys. Rev. B 85, 155139 (2012).

[48] Y.-M. Lin, X. Sun, and M. S. Dresselhaus, Phys. Rev. B 62, 4610 (2000).

[49] Y.-M. Lin, S. B. Cronin, J. Y. Ying, M. S. Dresselhaus, and J. P. Heremans, Appl. Phys. Lett. 76, 3944 (2000).

[50] G. Zhou, L. Li, and G. H. Li, J. Appl. Phys. 109, 114311 (2011). 